PROCEEDINGS OF THE

AMERICAN MATHEMATICAL SOCIETY

Volume 132, Number 2, Pages 597-606

S 0002-9939(03)07203-4

Article electronically published on August 20, 2003

\title{
ON THE GENUS OF ELLIPTIC FIBRATIONS
}

\author{
J.-B. GATSINZI \\ (Communicated by Paul Goerss)
}

\begin{abstract}
A simply connected topological space is called elliptic if both $\pi_{*}(X, \mathbb{Q})$ and $H^{*}(X, \mathbb{Q})$ are finite-dimensional $\mathbb{Q}$-vector spaces. In this paper, we consider fibrations for which the fibre $X$ is elliptic and $H^{*}(X, \mathbb{Q})$ is evenly graded. We show that in the generic cases, the genus of such a fibration is completely determined by generalized Chern classes of the fibration.
\end{abstract}

\section{INTRODUCTION}

In this paper, all topological spaces are supposed to be 1-connected and having the rational homotopy type of a $\mathrm{CW}$ complex of finite type.

The genus of a fibration $X \rightarrow E \stackrel{p}{\rightarrow} B$ is the least integer $n$ such that $B$ can be covered by $n+1$ open subsets, over each of which $p$ is a trivial fibration, in the sense of fibre homotopy type. We consider here the genus of fibrations whose fibres are elliptic spaces. For recall that a space $X$ is elliptic if both $H^{*}(X, \mathbb{Q})$ and $\pi_{*}(X) \otimes \mathbb{Q}$ are finite-dimensional $\mathbb{Q}$-vector spaces. Through this paper we work over rationals unless otherwise stated, and we will rely on the theory of Sullivan models.

We establish the following.

Theorem A. Let $X \rightarrow E \stackrel{p}{\rightarrow} B$ be a fibration where $X$ is a sphere. Such a fibration is classified by the map $f: B \rightarrow K(\mathbb{Q}, 2 k)$. Then the genus of $p$ is the nilpotency index of $\alpha=\operatorname{Im} H^{2 k}(f)$, that is, the least $r$ such that $\alpha^{r+1}=0$ (Theorem [2.3).

Theorem B. Given a fibration $X \rightarrow E \stackrel{p}{\rightarrow} B$ where $X$ is a homogeneous space $G / H$, when $G$ and $H$ have the same rank and $B$ is a formal space, the genus of $p$ is bounded above by nil $H^{\text {even }}(B)$ (Corollary 4.7).

In fact, we prove that the genus of $p$ is equal to the nilpotency index of the subalgebra of $H^{*}(B, \mathbb{Q})$ generated by the generalized Chern classes of the fibration.

\section{LS CATEGORY AND RELATED INVARIANTS}

Here we will recall some homotopy invariants of LS category type as well as the relation between the genus and universal fibrations.

Received by the editors October 6, 2001 and, in revised form, September 19, 2002.

2000 Mathematics Subject Classification. Primary 55P62; Secondary 55M30.

Key words and phrases. Rational homotopy, Lusternik-Schnirelmann category, genus, sectional category.

Supported by a grant from Université Catholique de Louvain.

(C)2003 American Mathematical Society 
Definition 1.1. The LS category of a space $X$, written cat $(X)$, is the least integer $n$ such that $X$ can be covered by $n+1$ open subsets, each contractible in $X$.

The original definition of the LS category differs from the one above by 1 (see [10]), but the definition above has become a standard in homotopy theory, since $\operatorname{cat}(X)=0$ if and only if $X$ is contractible. Since a direct computation of $\operatorname{cat}(X)$ is difficult, it is more convenient to approximate it by other invariants.

Definition 1.2. The nilpotency index of a $\operatorname{ring} R$, denoted by $\operatorname{nil}(R)$ is the least integer $n$ such that $R^{n+1}=0$. If $r \in R$, the nilpotency index of $r$ is the least $n$ such that $r^{n+1}=0$.

Note that in our definition, $\operatorname{nil}(R)$ is one unit less than the usual definition.

We have

$$
\operatorname{cat}(X) \geq \operatorname{nil} \tilde{H}(X)
$$

where $\tilde{H}$ is the reduced cohomology with any coefficient ring.

Definition 1.3. The category of a map $f: X \rightarrow Y$, denoted by cat $(f)$, is the least integer $n$ such that $X$ can be covered by $n+1$ open subsets $U_{i}$, for which the restriction of $f$ to each $U_{i}$ is nullhomotopic.

Note that

$$
\operatorname{cat}(f) \leq \min \{\operatorname{cat}(X), \operatorname{cat}(Y)\} .
$$

Moreover, $\operatorname{cat}(X)=\operatorname{cat}\left(i d_{X}\right)$, so that the category of a map is a generalisation of the LS category of a space. As in Equation (1), we have

$$
\operatorname{cat}(f) \geq \operatorname{nil}(\operatorname{Im} \tilde{H}(f))
$$

where

$$
\tilde{H}(f): \tilde{H}(Y) \rightarrow \tilde{H}(X)
$$

is the induced morphism in reduced cohomology with any coefficient ring.

Definition 1.4. Let $p: E \rightarrow B$ be a fibration. The sectional category of $p$, secat $(p)$, is the least integer $n$ such that $B$ can be covered by $n+1$ open subsets, over each of which $p$ has a section.

An approximation of $\operatorname{secat}(p)$ is given by the inequality [10]

$$
\operatorname{secat}(p) \geq \operatorname{nil}(\operatorname{ker} \tilde{H}(p)) \text {. }
$$

Definition 1.5. The genus of $p$ is the least integer $n$ such that $B$ can be covered by $n+1$ open subsets, over each of which $p$ is a trivial fibration, in the sense of fibre homotopy type.

It follows from the definitions above that

$$
\operatorname{secat}(p) \leq \operatorname{genus}(p),
$$

and equality holds if $p$ is a principal fibration.

If $f: B^{\prime} \rightarrow B$ is a map, consider $p^{\prime}: E^{\prime} \rightarrow B^{\prime}$, the fibration induced from $p$ by $f$. It is easily seen that secat $\left(p^{\prime}\right) \leq \operatorname{secat}(p)$, and equality holds if $f$ is a homotopy equivalence. The genus behaves in a similar way.

We define a similar invariant for $G$-bundles. If $p: E \rightarrow B$ is a $G$-bundle, define $\operatorname{Gcat}(p)$ as the least integer $n$ such that there is a covering of $B$ by $n+1$ open 
subsets over each of which $p$ is a trivial bundle. Of course, genus $(p) \leq \operatorname{Gcat}(p)$ and $\operatorname{Gcat}(p)=\operatorname{genus}(\pi)$, where $\pi$ is the associated principal fibre bundle. Moreover, if $f: B \rightarrow B G$ is the classifying map of $\pi$, then [10]

$$
\operatorname{Gcat}(p)=\operatorname{cat}(f) \text {. }
$$

In view of the relation above, if $p$ is a complex fibre bundle, then Chern classes may play a role in the estimation of the genus $(p)$. We will pursue this analogy for fibrations whose fibres are complex projective spaces.

As for Gcat, the genus is closely related to classifying spaces. Recall that fibrations with fibre in the homotopy type of $X$ are obtained, up to fibre homotopy equivalence, as a pull-back of the universal fibration [1]

$$
X \rightarrow B \text { aut }^{\bullet} X \rightarrow B \text { aut } X,
$$

where aut $X$ denotes the monoid of self-homotopy equivalences of $X$, aut $\boldsymbol{\theta}^{\bullet}$ is the monoid of pointed self-homotopy equivalences of $X$, and $B$ is the Dold-Lashof functor from monoids to topological spaces [2].

Letting $\tilde{B}$ aut $X \rightarrow B$ aut $X$ be the universal covering, the induced fibration $X \rightarrow \tilde{B}$ aut ${ }^{\bullet} X \rightarrow \tilde{B}$ aut $X$ is universal for fibrations with simply connected base spaces [4, Proposition 4.2].

The genus behaves like Gcat towards universal fibrations. We have

Theorem 1.6. [10] If $X \rightarrow E \stackrel{p}{\rightarrow} B$ is a fibration, then

$$
\operatorname{genus}(p)=\operatorname{cat}(f) \text {, }
$$

where $f: B \rightarrow B$ aut $X$ is the classifying map of $p$.

Some of the invariants above can also be defined in terms of existence of a section of a fibrewise join of fibrations. If $F_{1} \rightarrow E_{1} \stackrel{p_{1}}{\rightarrow} B$ and $F_{2} \rightarrow E_{2} \stackrel{p_{2}}{\rightarrow} B$ are fibrations with the same base, then the fibrewise join is the fibration $p_{1} * p_{2}: E_{1} *_{B} E_{2} \rightarrow B$, where elements of $E_{1} *_{B} E_{2}$ are of the form $\left(t_{1} e_{1}, t_{2} e_{2}\right), t_{1}+t_{2}=1, p_{1}\left(e_{1}\right)=$ $p_{2}\left(e_{2}\right)$, with the restriction that $t_{i} e_{i}$ is independent of $e_{i}$ if $t_{i}=0$. Naturally $\left(p_{1} * p_{2}\right)\left(t_{1} e_{1}, t_{2} e_{2}\right)=p_{1}\left(e_{1}\right)=p_{2}\left(e_{2}\right)$. Note that the fibre is the join $F_{1} * F_{2}$.

If $p$ is a fibration, $p(n)$ will denote the fibrewise join of $n+1$ copies of $p$. Consider the path fibration $\gamma: P B \rightarrow B$. The total space of the fibrewise join $\gamma(n)$ will be denoted by $G_{n}(B)$ and is often referred to as the $n$th Ganea space of $B$. The fibration $G_{n}(B) \rightarrow B$ is also called the Ganea fibration [7].

Theorem 1.7. Let $p: E \rightarrow B$ be a fibration and $\gamma: P B \rightarrow B$ the path fibration. $\operatorname{cat}(B)$ is the least integer $n$ such that $\gamma(n): G_{n}(B) \rightarrow B$ admits a section [7] and $\operatorname{secat}(p)$ is the least integer such that $p(n)$ has a section 10 .

In particular, for the path fibration $\gamma: P B \rightarrow B$, $\operatorname{cat}(B)=\operatorname{secat}(\gamma)=\operatorname{genus}(\gamma)$.

The category of a map can be defined using the Ganea fibration.

Theorem 1.8. 7] If $f: X \rightarrow Y$ is a mapping, cat $(f)$ is the least $n$ such that there is a lifting $\tilde{f}$ of $f$ in the following diagram:

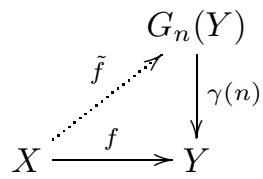


If one forms the pull-back

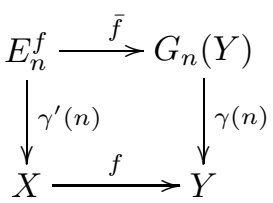

then $\operatorname{cat}(f)$ is the least $n$ such that the induced fibration $E_{n}^{f} \rightarrow X$ possesses a section.

The rational category of $X$, denoted by $\operatorname{cat}_{0}(X)$, is defined by $\operatorname{cat}_{0}(X)=$ $\operatorname{cat}\left(X_{0}\right)$. Here $X_{0}$ denotes the rationalization of $X$. For a mapping $f: X \rightarrow Y$, cat $_{0}(f)$ will denote cat $\left(f_{0}\right)$, where $f_{0}: X_{0} \rightarrow Y_{0}$ is the rationalization of $f$.

From now on, we will assume that $H^{i}(X)$ is a finite-dimensional $\mathbb{Q}$-vector space, for each $i$. Recall that the Sullivan minimal model of $X$ is a free commutative cochain algebra $(\Lambda Z, d)$ such that $d Z \subset \Lambda^{\geq 2} Z$, with $Z^{n} \cong \operatorname{Hom}_{\mathbb{Q}}\left(\pi_{n}(X), \mathbb{Q}\right)$ (see [16], 9]). Félix and Halperin showed that the rational category can be computed using Sullivan models, by exhibiting a model of the Ganea fibration $G_{n}(X) \rightarrow X$.

Theorem $1.9([\underline{5})$. Let $f: X \rightarrow Y$ be a mapping and $\bar{f}: \Lambda V \rightarrow \Lambda W$ its Sullivan minimal model. Then $\operatorname{cat}_{0}(f)$ is the least $n$ such that there is a mapping $\rho$ verifying $\bar{f}=\rho \circ i$ in the following diagram.

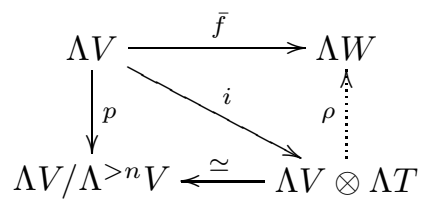

In particular, if $(\Lambda Z, d)$ is the Sullivan minimal model of $X$, then cat $_{0}(X)$ is the least integer $n$ such that $i$ has a retraction $\rho$.

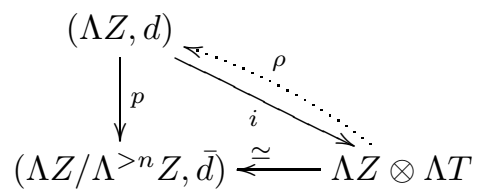

Since genus $(p)$ is the category of the classifying map, we recall here the construction of a model of $\tilde{B}$ aut $X$. If $(\Lambda Z, d)$ is a Sullivan model of $X$, then a Lie model of $\tilde{B}$ aut $X$ is obtained using derivations on $(\Lambda Z, d)$.

Precisely we define the differential Lie algebra $(\operatorname{Der} \Lambda Z, D)$ as follows [16]: in degree $k>1$, take the derivations of $\Lambda Z$ decreasing the degree by $k$. In degree one, we only consider the derivations $\theta$ that decrease the degree by one and verify $d \theta+\theta d=0$. The Lie bracket is defined by $\left[\theta, \theta^{\prime}\right]=\theta \theta^{\prime}-(-1)^{|\theta|\left|\theta^{\prime}\right|} \theta^{\prime} \theta$ and the differential $D$ is defined by $D \theta=[d, \theta]$.

Theorem 1.10 (16]). The graded differential Lie algebra $(\operatorname{Der} \Lambda Z, D)$ is a Lie model of $\tilde{B}$ aut $X$.

A model of $\tilde{B}$ aut $X$ from the Quillen model of $X$ is found in [13], 18], and [17. A Sullivan model of the universal fibration is given by the KS extension

$$
C^{*}(L) \rightarrow\left(C^{*}(L) \otimes \Lambda Z, D\right) \rightarrow(\Lambda Z, d)
$$


where $L=(\operatorname{Der} \Lambda Z, D)$. The explicit formula for $D$ is given in [18. Roughly speaking, for $z \in Z, D z=d z+\sum_{i} b_{i} \theta_{i}(z)$ where $\theta_{i}$ are those derivations vanishing on generators of degree greater than $|z|$ and the $b_{i}$ 's are their duals in $C^{*}(L)$.

\section{SPherical Fibrations}

We use Theorem 1.10 to compute a model of $\tilde{B}$ aut $X$, when $X$ is a sphere. We have the following.

Proposition 2.1. If $X=S^{2 n-1}$, then $(\tilde{B} \text { aut } X)_{0} \simeq K(\mathbb{Q}, 2 n)$ and if $X=S^{2 n}$, then $(\tilde{B} \text { aut } X)_{0} \simeq K(\mathbb{Q}, 4 n)$.

Proof. If $X=S^{2 n-1}$, then the Sullivan model of $X$ is $(\Lambda x, 0)$, where $|x|=2 n-1$. Hence

$$
\operatorname{Der}(\Lambda x, 0)=(\mathbb{Q} \cdot \alpha, 0)
$$

where $\alpha$ is the derivation taking $x$ to 1 . Hence $\tilde{B}$ aut $X$ has the rational homotopy type of $K(\mathbb{Q}, 2 n)$. For $X=S^{2 n}$, the Sullivan model is $(\Lambda(x, y), d)$ where $|x|=2 n$, $|y|=4 n-1, d x=0$, and $d y=x^{2}$. If $a$ is a generator of $\Lambda(x, y)$, let $(a, b)$ denote the derivation of $\Lambda(x, y)$ taking $a$ to $b$ and vanishing on the other generator. Here the Lie algebra $(L, \delta)=\operatorname{Der}(\Lambda(x, y), d)$ is generated (as a vector space) by the derivations

$$
\alpha_{2 n-1}=(y, x), \quad \alpha_{2 n}=(x, 1), \quad \alpha_{4 n-1}=(y, 1)
$$

and the differential is given by $\delta \alpha_{2 n-1}=\delta \alpha_{4 n-1}=0, \delta \alpha_{2 n}=2 \alpha_{2 n-1}$. Therefore $H_{i}(L, \delta)=\mathbb{Q}$ for $i=4 n-1$ and vanishes in all other degrees.

If $X=S^{2 n-1}$, a straightforward computation shows that a model of the universal fibration $X \rightarrow \tilde{B}$ aut $t^{\bullet} \rightarrow \tilde{B}$ aut $X$ is given by the KS extension

$$
\left(\Lambda y_{2 n}, 0\right) \rightarrow\left(\Lambda y_{2 n} \otimes \Lambda x_{2 n-1}, d\right) \rightarrow\left(\Lambda x_{2 n-1}, 0\right)
$$

where $d y_{2 n}=0, d x_{2 n-1}=y_{2 n}$. Since $\left(\Lambda y_{2 n} \otimes \Lambda x_{2 n-1}, d\right)$ is trivial, $X \rightarrow \tilde{B}$ aut $X \rightarrow$ $\tilde{B}$ aut $X$ is rationally equivalent to the path fibration. Therefore every fibration of fibre $S^{2 n-1}$ is rationally a principal fibration.

The sectional category of fibrations of fibre a sphere has been determined by D. Stanley, who proved, among other things, the following.

Theorem 2.2 ([15, Theorem 2.3]). Given a fibration $S^{2 n-1} \rightarrow E \stackrel{p}{\rightarrow} B$ with classifying map $f: B \rightarrow K(\mathbb{Q}, 2 n)$, if $\alpha=\operatorname{Im} H^{2 n}(f)$, then $\operatorname{secat}(p)=$ nil $\alpha$, that is, the least $r$ such that $\alpha^{r+1}=0$.

Since $(\tilde{B} \text { aut } X)_{0} \simeq K(\mathbb{Q}, 2 n)$ for $X=S^{2 n-1}$ and $(\tilde{B} \text { aut } X)_{0} \simeq K(\mathbb{Q}, 4 n)$ for $X=S^{2 n}$ (Proposition 2.1), we can generalize Stanley's result as follows.

Theorem 2.3. Let $X \rightarrow E \stackrel{p}{\rightarrow} B$ be a fibration such that $\tilde{B}$ aut $X$ is rationally homotopic to $K(\mathbb{Q}, 2 k)$. If $f: B \rightarrow K(\mathbb{Q}, 2 k)$ is the classifying map of $p$, then $\operatorname{genus}(p)=\operatorname{nil} \alpha$, where $\alpha=\operatorname{Im} H^{2 k}(f)$.

Under the hypotheses of Theorem [2.3], suppose that $X$ is an odd sphere. Then the resulting fibration is principal and $\operatorname{genus}(p)=\operatorname{secat}(p)=$ nil $\alpha$. We hence recover Theorem 2.2 
Proof of Theorem [2.3. The proof of Theorem 2.3 is based on the characterisation of $\operatorname{cat}(f)$ given by Theorem 1.9 Let $X \rightarrow E \stackrel{p}{\rightarrow} B$ be a fibration where $(\tilde{B} \text { aut } X)_{0} \simeq$ $K(\mathbb{Q}, 2 k)$ and $(\Lambda V, d) \rightarrow(\Lambda V \otimes \Lambda W, D) \rightarrow(\Lambda W, \bar{D})$ is a KS model of $p$. The KS extension above is classified by a mapping $f:(\Lambda z, 0) \rightarrow(\Lambda V, d)$ with $|z|=2 k$. Take $\alpha=[f(z)] \in H^{2 k}(\Lambda V, d)$. Suppose that $r$ is the smallest integer such that $\alpha^{r+1}=0$. Since cat $(f) \geq$ nil $\operatorname{Im} H(f)$ [10], we conclude that cat $(f) \geq r$.

On the other hand, consider the following diagram:

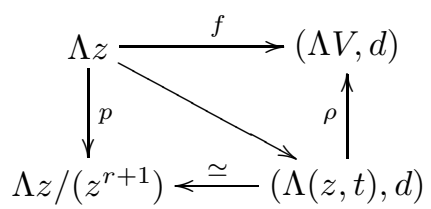

where $d t=z^{r+1}$. We define $\rho$ by $\rho(z)=f(z)$ and $\rho(t)=\beta$ where $d \beta=(f(z))^{r+1}$. Therefore $\operatorname{cat}(f)=\operatorname{genus}(p)=r$.

One can also prove Theorem 2.2 using the fibrewise join process. If $S^{2 n-1} \rightarrow$ $E \stackrel{p}{\rightarrow} B$ and $S^{2 m-1} \rightarrow E^{\prime} \stackrel{p^{\prime}}{\rightarrow} X$ are fibrations, then one can describe a model of $p * p^{\prime}$ as follows. Consider the KS extensions $\mathcal{B} \stackrel{\imath}{\rightarrow}(\mathcal{B} \otimes \Lambda a, d)$ and $\mathcal{B} \stackrel{\jmath}{\rightarrow}(\mathcal{B} \otimes \Lambda b, d)$ of $p$ and $p^{\prime}$ respectively. Note that $d a$ is a zero cohomology class in $H(\mathcal{B})$ if and only if $p$ is a trivial fibration. We use the method outlined by Doeraene in 3 to compute a model of the fibre join $p * p^{\prime}$. Consider the push-out

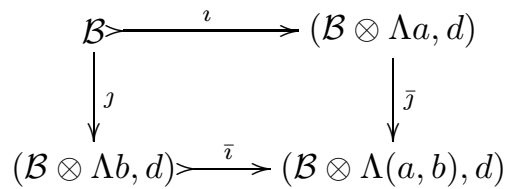

Since $\bar{\jmath}$ is not surjective, we form $(\mathcal{B} \otimes \Lambda a, d) \stackrel{\cong}{\rightrightarrows}(\mathcal{B} \otimes \Lambda(a, c, \bar{c}), d)$ with $d c=\bar{c}$ and $d \bar{c}=0$, which is a quasi-isomorphism. We define $f: \mathcal{B} \otimes \Lambda(a, c, \bar{c}) \rightarrow \mathcal{B} \otimes \Lambda(a, b)$ that extends $\bar{\jmath}$ by setting $f(c)=b$ and $f(\bar{c})=d b$.

Now we form the pull-back

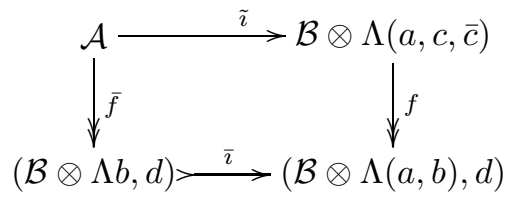

There is a natural mapping $\mathcal{B} \rightarrow \mathcal{A}$ that is a commutative model of $p * p^{\prime}$.

Recall that $\mathcal{A}=\{(x, y) \in(\mathcal{B} \otimes \Lambda b) \oplus(\mathcal{B} \otimes \Lambda(a, c, \bar{c})) \mid \bar{\imath}(x)=f(y)\}$. Hence a model of the join is the inclusion $b \mapsto(b, b)$. Since we know that the homotopic fibre of the fibrewise join is $S^{2 n-1} * S^{2 m-1}=S^{2(m+n)-1}$, its model is $\Lambda z$ where $|z|=|a|+|b|+1$. If $d a=\alpha$ and $d b=\beta$, then $(\alpha \beta, \alpha \beta) \in \mathcal{A}$ is a boundary in $\mathcal{A}$, since $d(\alpha b, \alpha c+a(\beta-\bar{c}))=(\alpha \beta, \alpha \beta)$. Therefore the relative Sullivan model of the fibre join is

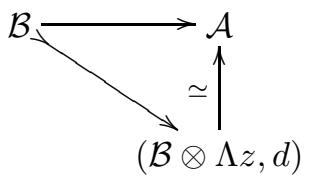


with $d z=\alpha \beta$. In particular, $p * p^{\prime}$ is a nontrivial fibration if and only if $\alpha \beta$ is a nonvanishing cohomology class in $H(\mathcal{B})$.

Working by induction, we can then deduce the following.

Proposition 2.4. Let $S^{2 n-1} \rightarrow E \stackrel{p}{\rightarrow} B$ be a fibration and $\mathcal{B} \rightarrow(\mathcal{B} \otimes \Lambda z, d)$ its $K S$-extension, where $d z=b \in \mathcal{B}$. Then a model of the $n$-fibrewise join $p(n)=$ $\underbrace{p * \cdots * p}_{(n+1) \text { factors }}$ is given by the $K S$-extension $\mathcal{B} \rightarrow(\mathcal{B} \otimes \Lambda w, d)$ with $d w=b^{n+1}$. In particular, $\operatorname{secat}(p)$ is the least $n$ such that $b^{n+1}$ is coboundary in $\mathcal{B}$ (see Theorem [2.2).

\section{The universal fibration of $\mathbb{C P}(n)$}

We consider here fibrations with fibre $\mathbb{C P}(n)$ of which the Sullivan model is $(\Lambda(a, b), d)$ with $d a=0$ and $d b=a^{n+1},|a|=2$ and $|b|=2 n+1$. To compute the rational homotopy type of $\tilde{B}$ aut $X$ we consider the derivations $\alpha_{2 i+1}=\left(b, a^{n-i}\right)$ of $\Lambda(a, b)$ for $i=0,1, \ldots, n$ and $\alpha_{2}=(a, 1)$ (subscripts indicate the degree). As a vector space, the Lie algebra $L$ of derivations of $(\Lambda(a, b), d)$ is

$$
L=\bigoplus_{i=0}^{n} \mathbb{Q} \alpha_{2 i+1} \oplus \mathbb{Q} \alpha_{2} .
$$

A straightforward computation shows that $\delta \alpha_{2 i+1}=0$ for all $0 \leq i \leq n$ and $\delta \alpha_{2}=(n+1) \alpha_{1}$. Hence for $1 \leq i \leq n, \alpha_{2 i+1}$ represents a nonzero homology class in $H_{*}(L, \delta)$. Therefore

$$
H_{*}(L, \delta)=\bigoplus_{i=1}^{n} \mathbb{Q} \alpha_{2 i+1}
$$

This implies that the Sullivan minimal model of $\tilde{B}$ aut $X$ is given by $\left(\Lambda\left(y_{4}, y_{6}, \ldots\right.\right.$, $\left.\left.y_{2 n+2}\right), 0\right)$ (see also [16, $\left.\S 11\right]$ ). Note that $\tilde{B}$ aut $X$ has the rational homotopy type of $\mathrm{B} S U(n+1)$.

A model for the universal fibration is given by the KS extension

$$
\left(\Lambda\left(y_{4}, y_{6}, \ldots, y_{2 n+2}\right), 0\right) \rightarrow\left(\Lambda\left(y_{4}, y_{6}, \ldots, y_{2 n+2}\right) \otimes \Lambda(a, b), D\right) \rightarrow(\Lambda(a, b), d)
$$

with

$$
D a=0, \quad D b=a^{n+1}+\sum_{i=0}^{n-1} a^{i} y_{2(n+1-i)} .
$$

Let $X \rightarrow E \stackrel{p}{\rightarrow} B$ be a fibration and $(\mathcal{B}, d)$ a Sullivan model of $B$. The KS model of $p$,

$$
\mathcal{B} \rightarrow(\mathcal{B} \otimes(a, b), D) \rightarrow(\Lambda(a, b), d),
$$

is classified by a mapping

$$
f:\left(\Lambda\left(y_{4}, y_{6}, \ldots y_{2 n+2}\right), 0\right) \rightarrow(\mathcal{B}, d) .
$$

Put $c_{4}=\left[f\left(y_{4}\right)\right], c_{6}=\left[f\left(y_{6}\right)\right], \ldots, c_{2 n+2}=\left[f\left(y_{2 n+2}\right)\right] \in H^{*}(\mathcal{B})$. We call $c_{4}, c_{6}, \ldots$, $c_{2 n+2}$ generalized Chern classes of the fibration $p$. Denote by $r_{4}, r_{6}, \ldots, r_{2 n+2}$ their respective nilpotency indexes, that is, $r_{k}$ is the least positive integer such that $c_{k}^{r_{k}+1}=0$.

We turn to fibrations for which the base is formal. For recall that a space $X$ is formal if there is a morphism $(\Lambda Z, d) \rightarrow H^{*}(\Lambda Z, d)$ that induces an isomorphism in cohomology, where $(\Lambda Z, d)$ is the Sullivan minimal model of $X$. 
Theorem 3.1. Let $\mathbb{C} P(n) \rightarrow E \stackrel{p}{\rightarrow} B$ be a fibration where $B$ is a formal space. The genus of $p$ is equal to the nilpotency index of $\operatorname{Im} H(f)$, where $f$ is the classifying map of $p$. In particular, if $m=\max \left\{r_{i}\right\}$ and $s=r_{4}+r_{6}+\cdots+r_{2 n+2}$, then $m \leq \operatorname{genus}(p) \leq s$.

Proof. Since $B$ is formal, there is a morphism $(\Lambda Z, d) \rightarrow H^{*}(\mathcal{B})$ inducing an isomorphism in homology. Consider the classifying map $f: \Lambda\left(y_{4}, y_{6}, \ldots, y_{2 n+2}\right) \rightarrow H^{*}(\mathcal{B})$. Let $k$ be the nilpotency index of $\operatorname{Im} f$. Since $\operatorname{cat}(f) \geq k$ by Equation 2 we need only to prove that cat $(f) \leq k$. It is then sufficient to check that $f$ factors through $\Lambda\left(y_{4}, y_{6}, \ldots, y_{2 n+2}\right) / \Lambda^{>k}\left(y_{4}, y_{6}, \ldots, y_{2 n+2}\right)$. If $t \in \Lambda^{>k}\left(y_{4}, y_{6}, \ldots, y_{2 n+2}\right)$, then $t$ is a finite sum of monomials of the form

$$
s y_{4}^{\beta_{4}} y_{6}^{\beta_{6}} \ldots y_{2 n+2}^{\beta_{2 n+2}}, \quad s \in \mathbb{Q}
$$

where $\beta_{i} \geq 0$ and $\sum \beta_{i}>k$. As a result, there is $i$ such that $\beta_{i} \geq r_{i}+1$. Therefore $f(t)=0$ and the result follows.

\section{Spaces Verifying the Halperin conjecture}

Definition 4.1. Let $X$ be an elliptic space. The integer

$$
\chi_{\pi}=\sum_{i}(-1)^{i} \operatorname{dim} \pi_{i}(X) \otimes \mathbb{Q}
$$

is called the homotopy Euler characteristic of $X$.

Theorem $4.2([8])$. If $X$ is an elliptic space, then the following statements are equivalent:

(1) $\chi_{\pi}=0$;

(2) $H^{*}(X, \mathbb{Q})$ is concentrated in even degrees.

Conjecture 4.3 (Halperin). Let $X \stackrel{i}{\rightarrow} E \stackrel{p}{\rightarrow} B$ be a fibration for which $X$ verifies one of the equivalent conditions of Theorem 4.2. Then the (rational) Serre spectral sequence collapses at the $E_{2}$ level or, equivalently, the morphism $H^{*}(i)$ : $H^{*}(E, \mathbb{Q}) \rightarrow H^{*}(X, \mathbb{Q})$ is surjective.

This conjecture has been verified in the following cases: if $H^{*}(X, \mathbb{Q})$ is generated by at most 3 generators [11, [19], if $X$ is a flag manifold [12, and if $X$ is a homogeneous space [14. In [12] Meier reformulated the conjecture in terms of homotopy groups of classifying spaces.

Theorem 4.4. Let $X$ be an elliptic space such that $H^{*}(X, \mathbb{Q})$ is concentrated in even degrees. The following statements are equivalent.

(1) The Serre spectral sequence for each fibration $X \stackrel{i}{\rightarrow} E \stackrel{p}{\rightarrow} B$ collapses at the $E_{2}$ level.

(2) There is no nonzero negative derivation on the algebra $H^{*}(X, \mathbb{Q})$.

(3) If $(\Lambda Z, d)$ is a Sullivan model of $X$, then $H_{*}(\operatorname{Der}(\Lambda Z, d))$ is concentrated in odd degrees.

(4) $\pi_{*}(\tilde{B}$ aut $X) \otimes \mathbb{Q}$ is concentrated in even degrees.

Example 4.5. The space $X$ of which a Sullivan commutative model is of the form $\Lambda x_{1} /\left(x_{1}^{n_{1}}\right) \otimes \cdots \otimes \Lambda x_{r} /\left(x_{r}^{n_{r}}\right)$, where $\left|x_{i}\right|$ is even, satisfies the Halperin conjecture. 
Consider a fibration of which the fibre is a homogeneous space $X=G / H$, $G$ and $H$ having the same rank. The Sullivan minimal model of $X$ is of the form $\left(\Lambda\left(x_{1}, \ldots, x_{r}, y_{1}, \ldots, y_{r}\right), d\right)$ where $\left|x_{i}\right|$ is even, $\left|y_{i}\right|$ is odd and $d y_{i}=f_{i} \in$ $\Lambda\left(x_{1}, \ldots, x_{r}\right)$. In [14], Shiga and Tezuka proved that this space verifies the Halperin conjecture, and hence

$$
L=H_{*}\left(\operatorname{Der}\left(\Lambda\left(x_{1}, \ldots, x_{r}, y_{1}, \ldots, y_{r}\right), d\right)\right)
$$

is concentrated in odd degrees. Hence the Lie bracket is trivial and $\tilde{B}$ aut $X$ has the rational homotopy type of a product of $K(\mathbb{Q}, 2 k)$. Take the derivations $\theta_{1}, \ldots, \theta_{n}$ representing homology classes in $L$. The Sullivan model of $\tilde{B}$ aut $X$ is then given by

$$
C^{*}(L)=\left(\Lambda\left(z_{1}, \ldots, z_{n}\right), 0\right),
$$

where the $z_{i}$ are of even degree and duals of $\theta_{i}$. We denote this model simply by $\Lambda Z$. A model of the universal fibration is given by

$$
\Lambda Z \rightarrow\left(\Lambda Z \otimes\left(x_{1}, \ldots, x_{r}, y_{1}, \ldots, y_{r}\right), D\right) \stackrel{p}{\rightarrow}\left(\Lambda\left(x_{1}, \ldots, x_{r}, y_{1}, \ldots, y_{r}\right), d\right)
$$

with $D y_{i}=d y_{i}+\sum_{j} z_{j} \theta_{j}\left(y_{i}\right)$ and $D x_{i}=0$ because $p$ is surjective in homology. Let $X \rightarrow E \rightarrow B$ be a fibration and

$$
(\mathcal{B}, d) \rightarrow\left(\mathcal{B} \otimes \Lambda\left(\left\{x_{i}, y_{i}\right\}\right), D\right) \rightarrow\left(\Lambda\left(\left\{x_{i}, y_{i}\right\}\right), d\right)
$$

its KS extension. We have the following push-out, where $f$ is the classifying map of the fibration $p$.

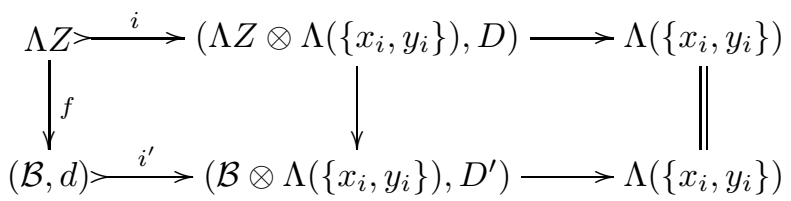

Moreover, $D^{\prime}\left(y_{i}\right)=f_{i}+\sum_{j} f\left(z_{j}\right) \theta_{j}\left(y_{i}\right)$, where $\left[f\left(z_{j}\right)\right] \in H^{*}(\mathcal{B}, d)$. If $B$ is formal, then cat $(f)=$ nil $H(f)$, and therefore the genus of $p$ is equal to the nilpotency index of the subalgebra of $H^{*}(\mathcal{B})$ generated by $\left\{f\left(z_{j}\right)\right\}$. Hence we get the following.

Proposition 4.6. Let $X \rightarrow E \stackrel{p}{\rightarrow} B$ be a rational fibration, where $B$ is a formal space and $X$ has the rational homotopy type of a homogeneous space $G / H, G$ and $H$ having the same rank. The genus of the rationalization of $p$ is equal to the nilpotency index of the subalgebra $\operatorname{Im}\left[H^{*}(\tilde{B}\right.$ aut $\left.X) \stackrel{\xi^{*}}{\rightarrow} H^{*}(B)\right]$ where $\xi: B \rightarrow \tilde{B}$ aut $X$ is the classifying map of $p$.

Corollary 4.7. Under the hypotheses of the above proposition, the genus of $p$ : $E \rightarrow B$ is bounded above by the nilpotency index of $H^{\text {even }}(B)$.

Remark 4.8. It is not clear how to obtain an upper bound of the sectional category using the index of nilpotency of some cohomology classes. Consider for instance the fibration $p$ of which the KS extension is

$$
\Lambda z_{2} \stackrel{i}{\rightarrow}\left(\Lambda z_{2} \otimes \Lambda\left(x_{2}, x_{5}\right), d\right) \quad \text { with } \quad d x_{5}=x_{2}^{3}-z_{2}^{3} .
$$

The mapping $i$ admits a retraction $r$ defined by $r\left(x_{2}\right)=r\left(z_{2}\right)=z_{2}, r\left(x_{5}\right)=0$. Hence the sectional category of $p$ is zero, while its genus is infinite by Proposition 3.1 


\section{REFERENCES}

[1] A. Dold, Halbexakte Homotopiefunktoren, Lecture Notes in Math., no. 12, Springer-Verlag, New York, 1966. MR 33:6622

[2] A. Dold and R. Lashoff, Principal quasi-fibrations and fibre homotopy equivalence of bundles, Illinois J. Math. 3 (1959), 285 - 305. MR 21:331

[3] J.-P. Doeraene, L.S.-category in a model category, J. Pure and Appl. Algebra 84 (1993), 215 - 261. MR 94b:55017

[4] E. Dror and A. Zabrodsky, Unipotency and nilpotency in homotopy equivalences, Topology 18 (1979), 187 - 197. MR 81g:55008

[5] Y. Félix, La dichotomie elliptique-hyperbolique en homotopie rationelle, Astérisque 176, Société Mathématique de France, 1989. MR 91c:55016

[6] Y. Félix and S. Halperin, Rational LS category and its applications, Trans. Amer. Math. Soc. 273 (1982), 1 - 37. MR 84h:55011

[7] T. Ganea, Lusternik-Schnirelmann category and strong category, Illinois J. Math. 11 (1967), $417-427$. MR 37:4814

[8] S. Halperin, Finiteness in the minimal models of Sullivan, Trans. Amer. Math. Soc. 230 (1977), 173 - 199. MR 57:1493

[9] S. Halperin, Lectures on minimal models, Mémoire de la Société Mathématique de France, $9-10,1983$. MR 85i:55009

[10] I. James, On category, in the sense of Lusternik-Schnirelmann, Topology 17 (1978), 331 348. MR 80i:55001

[11] G. Lupton, Note on a conjecture of Stephen Halperin, Lecture Notes in Math., no. 1440, Springer-Verlag, New York, 1990, 148 - 163. MR 92a:55012

[12] W. Meier, Rational universal fibrations and flag manifolds, Math. Ann. 258 (1981/82), 329 340. MR 83g:55009

[13] M. Schlessinger and J. Stasheff, Deformations theory and rational homotopy type, preprint.

[14] H. Shiga and M. Tezuka, Rational fibrations, homogeneous spaces with positive Euler characteristics and Jacobians, Ann. Inst. Fourier 37 (1987), 81 - 106. MR 89g:55019

[15] D. Stanley, The sectional category of spherical fibrations, Proc. Amer. Math. Soc. 128 (2000), 3137 - 3143. MR 2001a:55004

[16] D. Sullivan, Infinitesimal computations in topology, Inst. Hautes Études Sci. Publ. Math. 47 (1977), 269 - 331. MR 58:31119]

[17] D. Tanré, Homotopie rationnelle: Modèles de Chen, Quillen, Sullivan, Lecture Notes in Math., no. 1025, Springer-Verlag, Berlin, 1983. MR 86b:55010

[18] D. Tanré, Fibrations et Classifiants, In Homotopie algébrique et algèbre locale, Astérisque 113 - 114, 1984. MR 85h:55018

[19] J. C. Thomas, Rational homotopy of Serre fibrations, Ann. Inst. Fourier (Grenoble) 31 (1981), 71 - 90. MR 83c:55016

University of Botswana, Private Bag 0022, Gaborone, Botswana

E-mail address: gatsinzj@mopipi.ub.bw 